\title{
Type 1 Diabetic Subjects with Diabetic Retinopathy Show an Unfavorable Pattern of Fat Intake
}

\author{
Minerva Granado-Casas 1,2 (iD), Anna Ramírez-Morros ${ }^{1}$, Mariona Martín ${ }^{3}$, Jordi Real 4,5,6,
}

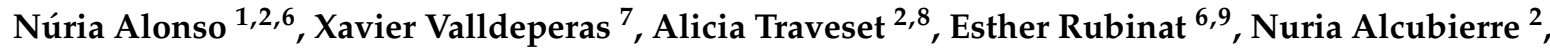
Marta Hernández ${ }^{2,10}$, Manel Puig-Domingo ${ }^{1,6}$, Albert Lecube ${ }^{2,10}$, Esmeralda Castelblanco ${ }^{1,6, *}$ and Didac Mauricio $2,6,11, *$ (I)

1 Department of Endocrinology and Nutrition, Health Sciences Research Institute \& University Hospital Germans Trias i Pujol, 08916 Badalona, Spain; mgranado@igtp.cat (M.G.-C.); aramirez@igtp.cat (A.R.-M.); nalonso32416@yahoo.es (N.A.); mpuigd@igtp.cat (M.P.-D.)

2 Lleida Institute for Biomedical Research Dr. Pifarré Foundation, IRBLleida, University of Lleida, 25198 Lleida, Spain; atravesetm@gmail.com (A.T.); cubito@lleida.org (N.A.);

martahernandezg@gmail.com (M.H.); alecube@gmail.com (A.L.)

3 Department of Endocrinology \& Nutrition, University Hospital Germans Trias i Pujol, 08916 Badalona, Spain; marionamarting@gmail.com

4 Epidemiology and Public Health, International University of Catalonia, 08017 Barcelona, Spain; jordireal@gmail.com

5 Unit Support of Research, Institut d'Investigació en Atenció Primària Jordi Gol (IDIAP Jordi Gol), 08007 Barcelona, Spain

6 Center for Biomedical Research on Diabetes and Associated Metabolic Diseases (CIBERDEM), Instituto de Salud Carlos III, 08907 Barcelona, Spain; rubinatesther@gmail.com

7 Department of Ophthalmology, University Hospital Germans Trias i Pujol, 08916 Badalona, Spain; xvalldeperas@gmail.com

8 Department of Ophthalmology, University Hospital Arnau de Vilanova, 25198 Lleida, Spain

9 Department of Nursing and Physiotherapy, University of Lleida, 25198 Lleida, Spain

10 Department of Endocrinology \& Nutrition, University Hospital Arnau de Vilanova, 25198 Lleida, Spain

11 Department of Endocrinology \& Nutrition, Hospital de la Santa Creu i Sant Pau, Autonomous University of Barcelona, 08041 Barcelona, Spain

* Correspondence: esmeraldacas@gmail.com (E.C.); didacmauricio@gmail.com (D.M.); Tel.: +34-934-978-655 (E.C.); +34-935-565-661 (D.M.)

Received: 13 July 2018; Accepted: 27 August 2018; Published: 29 August 2018

\begin{abstract}
Medical nutrition therapy is an important part of the management of type 1 diabetes mellitus (T1DM). Proper adherence to a healthy diet may have a favorable impact on diabetes management and its diabetic complications. Our aim was to assess differences in food and nutrient intake of type 1 diabetic patients with and without diabetic retinopathy (DR). This was a two-center, cross-sectional study in patients with T1DM, with and without DR. Subjects were recruited from the outpatient clinic of the two participating centers. A validated food frequency questionnaire was administered. A total of 103 T1DM patients with DR and 140 T1DM patient without DR were recruited. Subjects with DR showed a lower intake of total fat $(p=0.036)$ than that of their non-DR counterparts. DR was associated with increasing age $(p=0.004)$, hypertension $(p<0.001)$, and diabetes duration $(p<0.001)$, however there was a negative association with high educational level $(p=0.018)$. The multivariate-adjusted analysis showed that the intake of complex carbohydrates was positively related to the presence of DR $(p=0.031)$. In contrast, the intakes of total fat $(p=0.009)$, monounsaturated fatty acids (MUFAs) $(p=0.012)$, oleic acid $(p=0.012)$, and vitamin $\mathrm{E}(p=0.006)$ were associated with the absence of DR. As conclusions, the intake of total MUFAs, oleic acid, and vitamin $E$ is associated with a lower frequency of DR in patients with T1DM. These results suggest a potential protective effect of these lipid components for DR.
\end{abstract}


Keywords: type 1 diabetes; diabetic retinopathy; dietary pattern; oleic acid; fatty acids; carbohydrates

\section{Introduction}

Diabetic retinopathy (DR) is an important cause of visual impairment in patients with type 1 diabetes mellitus (T1DM) [1]. According to a previous study in a cohort of patients with T1DM, the 10-year incidence of DR was 35.9\% [2]. The American Diabetes Association (ADA) and European Association for the Study of Diabetes (EASD) establish that medical nutrition therapy (MNT) plays an important role in preventing complications related to the management and metabolic control of the disease [3]. These nutritional recommendations are based on the Mediterranean Diet, which has benefits to prevent cardiovascular diseases in the diabetic population. Additionally, a positive relationship between severity of DR and modifiable risk factors such as hypertension, dyslipidemia, and smoking has been described in T1DM [1].

Currently, a controversy exists with the few published studies, regarding the relationship between the dietary intake and the presence of DR in patients with diabetes, describing controversial results between them $[4,5]$. The relationship between dietary intake and DR development in patients with T1DM has only been described in few studies [6-12]; specifically, there are only seven studies that have assessed the relationship between dietary intake and the prevalence of DR in patients with T1DM [6-12]. Of these, four studies related alcohol consumption and/or salt intake with the frequency of DR in a cross-sectional study design [9-12]. Results of the Diabetes Control and Complications Trial (DCCT) study cohort pointed to higher risk of DR progression in those subjects with higher intake of fatty acids, and a lower-risk association among those with higher dietary fiber intake [6]; however, another study did not find any differences [7]. A study with a cross-sectional design and a sample of 379 patients with diabetes mellitus (of whom only 52 had T1DM) observed a protective effect of MUFA and polyunsaturated fatty acid (PUFA) intake for DR in well-controlled patients [8]. The EURODIAB Study only examined the relationship between alcohol consumption and salt intake with the risk of DR in T1DM patients [9,10]. They found a lower risk of DR with a moderate alcohol consumption [9]; nevertheless, no association was observed with an increased salt intake with DR in this diabetic population [10]. On the other hand, the FinnDiane Study [11] and Moss et al. [12] found an inverse association between alcohol consumption and the presence of DR in these patients. According to two recently published systematic reviews about the relationship between dietary intake and DR, no study has assessed the relationship between the whole food and nutrient intake and DR in patients with T1DM [4,5].

In our region, apart from receiving specific education on carbohydrate counting learning, medical nutritional therapy for diabetic subjects includes recommendations to adopt a Mediterranean Diet pattern $[13,14]$. To our knowledge, there are no studies designed to compare the relationship between the food and nutrient intake in T1DM patients with the presence or absence of DR. In addition, our group described a positive association between the higher intake of MUFAs and oleic acid and a lower frequency of DR in patients with T2DM [15]. For this reason, we hypothesized that patients with T1DM and DR have a lower adherence to the nutritional recommendations than that of their counterparts without DR. Therefore, the aim of the current study was to assess differences in the food and nutrient intake of patients with T1DM, with and without DR.

\section{Materials and Methods}

This was a cross-sectional, two-center study. The participants were regularly cared for at their reference hospital (University Hospital Arnau de Vilanova and University Hospital Germans Trias i Pujol, both in Northeastern Spain). The study participants were recruited from the health care districts of Lleida (Center 1) and Badalona (Center 2). Lleida is a rural and semi-urban region, while Badalona is an urban location in the metropolitan area of Barcelona. This was a post hoc analysis from a previously 
published study [14]; from a sample of 259 patients with T1DM, 16 patients were excluded because they did not have a full assessment of DR status. A final sample of 103 T1DM patients with DR and 140 T1DM without DR was included. More details of the original study are available in [14]. The recruitment was performed between January 2013 and May 2015. As inclusion criteria, we used the following: T1DM with a duration of at least one year and age over 18 years. The exclusion criteria were as follows: patients who were healthcare professionals; patients who showed physical or cognitive deterioration (e.g., dementia, mental diseases); history of clinical cardiovascular disease or diabetic foot disease, pregnancy, renal insufficiency (estimated glomerular filtration rate $<60 \mathrm{~mL} / \mathrm{min}$ ); and patients with a condition that required additional MNT measures (i.e., macroalbuminuria defined as urine albumin/creatinine ratio $>299 \mathrm{mg} / \mathrm{g}$ ).

The ethics committees of the two participating centers approved the study, and written informed consent forms were obtained from all participants (PI-13-095 and PI-15-147).

\subsection{Clinical Variables}

Clinical variables were collected through detailed anamnesis, physical exam, and careful review of the clinical records. Hypertension and dyslipidemia were defined by the use of any specific medication for the given conditions. Blood and urine chemistry variables were determined by standardized methods. Physical activity was assessed by the validated method of Bernstein et al. [16] and Cabrera de León et al. [17]. A subject was classified as having regular physical activity if she/he performed more than $25 \mathrm{~min} /$ day of any type of physical activity that requires 4 METS (the metabolic equivalent) minimum. Participants were classified as sedentary if they did less than $25 \mathrm{~min} /$ day of any physical activity.

\subsection{Dietary and Nutritional Assessment}

A food frequency questionnaire (FFQ) was administered to all participants of the study during personal interviews by specialized and trained researchers. This was a semi-quantitative questionnaire, validated and adapted for the Spanish population, based on the Nurses' Health Study (available at: http:/ / epinut.edu.umh.es/wp-content/uploads/sites/1365/2011/07/CFA101. pdf) $[18,19]$. The questionnaire contained 101 items, in which it asked for their usual consumption since the previous year's visit. Serving sizes were detailed for each food item in the FFQ. The questionnaire had nine possible answers, ranging from "never or less than once per month" to "six or more per day". Nutrient values were primarily obtained from the food composition tables of the US Department of Agriculture and other published sources for Spanish and English foods and portion sizes [20-22]. We used the Spanish food composition tables to avoid an overestimation of nutrient intake from the fortified dairy products of the US.

\subsection{Assessment of Diabetic Retinopathy}

Retinopathy was assessed and classified by an ophthalmologist according to the international clinical classification system [23]. The ophthalmologist performed a complete eye evaluation and defined DR in five stages: (1) no apparent retinopathy, (2) mild non-proliferative retinopathy, (3) moderate non-proliferative retinopathy, (4) severe non-proliferative retinopathy, and (5) proliferative diabetic retinopathy.

\subsection{Statistical Analysis}

Descriptive comparison between groups of all variables was performed to evaluate the differences. Statistical significance was assessed by the chi-square test, where the frequencies of comorbidities and demographic variables were compared among groups. The mean differences between groups were compared by Student's $t$-test. Methods of Benjamini, Hochberg, and Yekutieli were performed to control the false discovery rate and expected proportion of false discoveries among the rejected hypotheses. Conditional logistic regression models were developed to analyze the relationship 
between the presence of diabetic retinopathy (DR), its clinical characteristics, and daily nutrient intake. Crude and adjusted odds ratios (ORs) were estimated by univariable and multivariable conditional logistic regression models, respectively. Adjusted odds ratios were fitted by age, sex, educational level, smoking, center, physical activity, body mass index, dyslipidemia, hypertension, and diabetes duration. In all models, the goodness-of-fit assumption was tested by the Hosmer-Lemeshow test. Estimates of odds ratios were reported with corresponding 95\% confidence intervals and statistical significance was established as a $p$-value $<0.05$. Data management and analyses were performed with the free software environment R version 3.3.2 and SPSS software (version 20, SPSS, Chicago, IL, USA).

\section{Results}

Clinical characteristics of the participants are shown in Table 1. Patients with DR demonstrated the following: were older $(p=0.010)$, had higher systolic blood pressure $(p=0.005)$ and frequency of hypertension $(p<0.001)$, had longer duration of diabetes $(p<0.001)$ and showed worse glycemic control $(p<0.001)$ and lipid profile, and had lower HDL $(p=0.045)$ and higher triglycerides $(p=0.045)$.

Table 1. Clinical characteristics of participants according to diabetic retinopathy status.

\begin{tabular}{|c|c|c|c|}
\hline Characteristics & DR $(n=103)$ & No DR $(n=140)$ & $p^{1}$ \\
\hline Age (years) & $46.2 \pm 10.8$ & $42.1 \pm 10.3$ & 0.010 \\
\hline Sex (male) & $48(46.6)$ & $62(44.3)$ & 0.867 \\
\hline Educational level & & & 0.107 \\
\hline Not even primary & $4(4.1)$ & $8(5.9)$ & \\
\hline Completed primary & $36(36.0)$ & $34(25.2)$ & \\
\hline Secondary high school & $39(40.2)$ & $49(36.3)$ & \\
\hline Graduate or higher & $18(18.6)$ & $44(32.6)$ & \\
\hline Smoking & & & 0.602 \\
\hline No & $48(46.6)$ & $73(52.1)$ & \\
\hline Yes & $32(31.1)$ & $34(24.3)$ & \\
\hline Former smoker & $23(22.3)$ & $33(23.6)$ & \\
\hline Regular physical activity & $77(74.8)$ & $98(70.0)$ & 0.602 \\
\hline Waist circumference $(\mathrm{cm})$ & $90.7 \pm 13.4$ & $87.0 \pm 12.1$ & 0.075 \\
\hline Systolic blood pressure $(\mathrm{mmHg})$ & $131.0 \pm 18.4$ & $123.2 \pm 16.1$ & 0.005 \\
\hline Diastolic blood pressure ( $\mathrm{mmHg}$ ) & $73.8 \pm 9.3$ & $74.2 \pm 9.6$ & 0.846 \\
\hline BMI $\left(\mathrm{kg} / \mathrm{m}^{2}\right)$ & $26.2 \pm 4.3$ & $25.3 \pm 4.0$ & 0.111 \\
\hline Hypertension & $40(38.8)$ & $20(14.3)$ & $<0.001$ \\
\hline Dyslipidemia & $49(47.6)$ & $49(35.0)$ & 0.107 \\
\hline Diabetes duration (years) & $26.5 \pm 9.9$ & $17.9 \pm 9.1$ & $<0.001$ \\
\hline $\operatorname{HbA} 1 \mathrm{c}(\%)$ & $7.9 \pm 1.1$ & $7.4 \pm 0.8$ & $<0.001$ \\
\hline $\mathrm{HbA} 1 \mathrm{c}(\mathrm{mmol} / \mathrm{mol})$ & $63.0 \pm 11.9$ & $56.9 \pm 8.3$ & $<0.001$ \\
\hline Total cholesterol (mg/dL) & $179.0 \pm 30.8$ & $182.3 \pm 27.1$ & 0.602 \\
\hline HDL cholesterol (mg/dL) & $61.9 \pm 16.8$ & $66.8 \pm 14.9$ & 0.045 \\
\hline LDL cholesterol (mg/dL) & $102.7 \pm 25.5$ & $102.2 \pm 22.7$ & 0.993 \\
\hline $\mathrm{TG}(\mathrm{mg} / \mathrm{dL})$ & $81.4 \pm 49.9$ & $68.7 \pm 26.7$ & 0.045 \\
\hline
\end{tabular}

Data are means $\pm \mathrm{SD}$ or $\mathrm{n}(\%) .{ }^{1} p$ was calculated by method of Benjamini and Hochberg. DR, diabetic retinopathy; $\mathrm{HbA1c}$, glycated hemoglobin; BMI, body mass index; HDL cholesterol, high density lipoprotein cholesterol; LDL cholesterol, low density lipoprotein cholesterol; TG, triglycerides.

Concerning daily nutrient intake, patients with DR had a lower fat intake than those without DR did ( $p=0.036$ ) (Table 2). Although not significantly different, the intake of MUFAs, oleic acid, and vitamin $\mathrm{E}$ was lower in patients with DR than in those without $(p=0.050)$. In terms of daily food intake, no statistical differences were found between patients with and without DR (Table S1). However, a tendency to eat more bread $(p=0.072)$ and less vegetable fat $(p=0.072)$ was observed in patients with DR. 
Table 2. Daily nutrient intake of participants according to diabetic retinopathy status.

\begin{tabular}{|c|c|c|c|}
\hline Daily Nutrient Intake $^{1}$ & $\mathrm{DR}(n=103)$ & No DR $(n=140)$ & $p^{2}$ \\
\hline Energy intake (kcal) & $2047.0 \pm 556.0$ & $2077.7 \pm 482.3$ & 0.796 \\
\hline Carbohydrate (g) & $198.0 \pm 36.3$ & $186.6 \pm 31.2$ & 0.075 \\
\hline Complex carbohydrate (g) & $91.6 \pm 18.6$ & $85.8 \pm 18.1$ & 0.096 \\
\hline Sugar $(\mathrm{g})$ & $82.0 \pm 24.3$ & $78.9 \pm 23.1$ & 0.604 \\
\hline Fiber $(\mathrm{g})$ & $23.4 \pm 5.8$ & $22.4 \pm 6.2$ & 0.545 \\
\hline Soluble fiber (g) & $3.6 \pm 1.3$ & $3.5 \pm 1.2$ & 0.604 \\
\hline Insoluble fiber (g) & $13.6 \pm 4.4$ & $12.9 \pm 4.1$ & 0.545 \\
\hline Glycemic index (\%) & $87.0 \pm 18.1$ & $81.1 \pm 15.8$ & 0.075 \\
\hline Protein $(\mathrm{g})$ & $98.7 \pm 16.0$ & $97.0 \pm 14.0$ & 0.604 \\
\hline Total fat (g) & $98.9 \pm 16.3$ & $105.8 \pm 13.9$ & 0.036 \\
\hline SFAs (g) & $25.4 \pm 5.1$ & $26.8 \pm 4.4$ & 0.193 \\
\hline MUFAs (g) & $50.2 \pm 11.2$ & $54.3 \pm 9.9$ & 0.050 \\
\hline PUFAs (g) & $16.5 \pm 4.1$ & $17.8 \pm 5.7$ & 0.193 \\
\hline Omega $3(\mathrm{~g})$ & $1.6 \pm 0.4$ & $1.7 \pm 0.4$ & 0.263 \\
\hline Omega $6(\mathrm{~g})$ & $14.8 \pm 4.1$ & $16.0 \pm 5.8$ & 0.214 \\
\hline Trans fat (g) & $1.0 \pm 0.5$ & $1.0 \pm 0.5$ & 0.864 \\
\hline Cholesterol (mg) & $290.0 \pm 75.4$ & $287.7 \pm 66.6$ & 0.834 \\
\hline Palmitic acid (g) & $15.7 \pm 2.7$ & $16.5 \pm 2.2$ & 0.090 \\
\hline Stearic acid (g) & $5.9 \pm 1.2$ & $6.1 \pm 1.1$ & 0.545 \\
\hline Oleic acid (g) & $47.7 \pm 11.0$ & $51.7 \pm 9.7$ & 0.050 \\
\hline Linoleic acid (g) & $14.7 \pm 4.1$ & $15.9 \pm 5.8$ & 0.214 \\
\hline$\alpha$-Linolenic acid (g) & $1.1 \pm 0.2$ & $1.2 \pm 0.2$ & 0.214 \\
\hline Arachidonic acid (g) & $0.2 \pm 0.1$ & $0.2 \pm 0.0$ & 0.604 \\
\hline EPA $(g)$ & $0.2 \pm 0.1$ & $0.2 \pm 0.1$ & 0.604 \\
\hline DHA (g) & $0.3 \pm 0.2$ & $0.3 \pm 0.2$ & 0.604 \\
\hline Alcohol (g) & $6.5 \pm 11.6$ & $5.6 \pm 9.2$ & 0.686 \\
\hline Caffeine (g) & $244.0 \pm 196.0$ & $275.4 \pm 233.9$ & 0.601 \\
\hline Water (g) & $2962.0 \pm 720.0$ & $2883.5 \pm 676.8$ & 0.604 \\
\hline Vitamin A $(\mu \mathrm{g})$ & $1283.0 \pm 671.0$ & $1212.1 \pm 705.7$ & 0.604 \\
\hline Retinol $(\mu \mathrm{g})$ & $355.0 \pm 464.0$ & $346.9 \pm 374.3$ & 0.887 \\
\hline Carotene $(\mu \mathrm{g})$ & $900.0 \pm 436.0$ & $841.6 \pm 547.1$ & 0.604 \\
\hline$\alpha$ Carotene $(\mu \mathrm{g})$ & $703.0 \pm 535.0$ & $610.2 \pm 581.2$ & 0.545 \\
\hline$\beta$ Carotene $(\mu \mathrm{g})$ & $4868.0 \pm 2324.0$ & $4578.6 \pm 2957.4$ & 0.604 \\
\hline B Cryptoxanthin $(\mu \mathrm{g})$ & $332.0 \pm 222.0$ & $302.0 \pm 190.4$ & 0.602 \\
\hline Lutein+zeoxanthin $(\mu \mathrm{g})$ & $4147.0 \pm 2479.0$ & $3951.2 \pm 360.3$ & 0.796 \\
\hline Lycopene $(\mu \mathrm{g})$ & $4457.0 \pm 2096.0$ & $4292.6 \pm 2065.8$ & 0.658 \\
\hline Folate $(\mu \mathrm{g})$ & $292.0 \pm 73.0$ & $283.7 \pm 81.7$ & 0.604 \\
\hline Vitamin $B_{12}(\mathrm{mg})$ & $8.3 \pm 3.8$ & $8.5 \pm 3.5$ & 0.796 \\
\hline Vitamin $B_{6}(\mathrm{mg})$ & $1.9 \pm 0.5$ & $1.9 \pm 0.5$ & 0.802 \\
\hline Vitamin C (mg) & $116.0 \pm 62.4$ & $112.3 \pm 55.6$ & 0.796 \\
\hline Vitamin D (mg) & $4.3 \pm 1.7$ & $4.4 \pm 1.6$ & 0.828 \\
\hline Vitamin E (mg) & $13.9 \pm 2.9$ & $15.2 \pm 3.6$ & 0.050 \\
\hline Thiamine (mg) & $1.6 \pm 0.2$ & $1.5 \pm 0.3$ & 0.075 \\
\hline Riboflavin (mg) & $2.3 \pm 0.5$ & $2.2 \pm 0.5$ & 0.796 \\
\hline Niacin (mg) & $27.9 \pm 6.0$ & $27.8 \pm 6.3$ & 0.860 \\
\hline Niacin equivalents (mg) & $44.0 \pm 8.0$ & $43.6 \pm 7.8$ & 0.796 \\
\hline Calcium (mg) & $1115.0 \pm 329.0$ & $1108.2 \pm 317.7$ & 0.887 \\
\hline Iron (mg) & $13.1 \pm 2.2$ & $13.0 \pm 2.8$ & 0.796 \\
\hline Sodium (mg) & $3381.0 \pm 520.0$ & $3449.8 \pm 579.7$ & 0.604 \\
\hline Potassium (mg) & $3491.0 \pm 695.0$ & $3332.5 \pm 652.4$ & 0.262 \\
\hline Magnesium (mg) & $415.0 \pm 77.4$ & $405.4 \pm 84.4$ & 0.604 \\
\hline Zinc (mg) & $11.7 \pm 1.7$ & $11.4 \pm 1.5$ & 0.561 \\
\hline Selenium ( $\mu \mathrm{g})$ & $145.0 \pm 26.6$ & $142.1 \pm 24.0$ & 0.604 \\
\hline
\end{tabular}

${ }^{1}$ Adjusted by energy intake. Data are means \pm SD. ${ }^{2} p$ was calculated by method of Benjamini and Hochberg. DR, diabetic retinopathy; SFAs, saturated fatty acids; MUFAs, monounsaturated fatty acids; PUFAs, polyunsaturated fatty acids; EPA, eicosapentaenoic acid; DHA, docosahexaenoic acid. 
In the bivariate analysis, including the clinical characteristics (Table 3), higher educational level was associated with the absence of DR (OR: 0.47; 95\% CI: $0.25-0.88 ; p=0.018)$. In contrast, DR was associated with increasing age (OR: 1.04; 95\% CI: 1.01-1.06; $p=0.004)$, hypertension (OR: 3.77; 95\% CI: 2.05-7.13; $p<0.001$ ), diabetes duration (OR: 1.10; 95\% CI: 1.07-1.14; $p<0.001$ ), and glycemic control (OR: 1.97, 95\% CI: 1.44-2.69; $p=0.001)$.

Table 3. Bivariate analysis for the relationship between the presence of diabetic retinopathy and clinical characteristics.

\begin{tabular}{ccccc}
\hline Characteristics & DR $(\boldsymbol{n}=\mathbf{1 0 3})$ & No DR $(\boldsymbol{n = 1 4 0 )}$ & OR $[\mathbf{9 5} \% \mathbf{C I}]^{\mathbf{1}}$ & $p$ \\
\hline Sex (male) & $48(46.6)$ & $62(44.3)$ & $1.10[0.66 ; 1.83]$ & 0.722 \\
Completed primary & $36(36.0)$ & $34(25.2)$ & $1.75[1.00 ; 3.09]$ & 0.052 \\
Secondary high school & $39(40.2)$ & $49(36.3)$ & $1.17[0.69 ; 1.99]$ & 0.545 \\
Graduate or higher & $18(18.6)$ & $44(32.6)$ & $0.47[0.25 ; 0.88]$ & 0.018 \\
Smoker, current & $32(31.1)$ & $34(24.3)$ & $1.35[0.76 ; 2.38]$ & 0.241 \\
Smoker, former & $23(22.3)$ & $33(23.6)$ & $0.95[0.52 ; 1.73]$ & 0.820 \\
Site Lleida & $51(49.5)$ & $55(39.3)$ & $1.60[0.96 ; 2.67]$ & 0.113 \\
Physical activity & $77(74.8)$ & $98(70.0)$ & $1.27[0.72 ; 2.27]$ & 0.420 \\
Age (years) & $46.2 \pm 10.8$ & $42.1 \pm 10.3$ & $1.04[1.01 ; 1.06]$ & 0.004 \\
BMI (kg/m $\left.{ }^{2}\right)$ & $26.2 \pm 4.3$ & $25.3 \pm 4.0$ & $1.06[0.99 ; 1.13]$ & 0.073 \\
Dyslipidemia & $49(47.6)$ & $49(35.0)$ & $1.68[1.00 ; 2.84]$ & 0.050 \\
Hypertension & $40(38.8)$ & $20(14.3)$ & $3.77[2.05 ; 7.13]$ & $<0.001$ \\
Diabetes duration (years) & $26.5 \pm 9.9$ & $17.9 \pm 9.1$ & $1.10[1.07 ; 1.14]$ & $<0.001$ \\
HbA1c (\%) & $7.9 \pm 1.1$ & $7.4 \pm 0.8$ & $1.97[1.44 ; 2.69]$ & 0.001 \\
\hline
\end{tabular}

Data are mean \pm SD or $n(\%) .{ }^{1}$ Odds ratio and $95 \%$ confidence interval of diabetic retinopathy by clinical characteristics. DR, diabetic retinopathy; BMI, body mass index; HbA1c, glycated hemoglobin.

In the crude multivariate analysis for nutrient intake, a high intake of complex carbohydrates ( $p=0.031$ ) was associated with the presence of DR (Table 4$)$. On the other hand, the absence of retinopathy was associated with a higher intake of total fat $(p=0.009)$, MUFAs $(p=0.012)$, oleic acid $(p=0.012)$, and vitamin $\mathrm{E}(p=0.006)$. In the multivariate adjusted model, only the intake of complex carbohydrates $(p=0.031)$ remained associated with the presence of DR, whereas the intake of total fat $(p=0.009)$, MUFAs $(p=0.012)$, oleic acid $(p=0.012)$, and vitamin $\mathrm{E}(p=0.006)$ were still associated with the absence of DR.

Table 4. Crude and adjusted multivariate analysis for the presence of diabetic retinopathy and daily nutrient intake.

\begin{tabular}{|c|c|c|c|c|c|c|}
\hline Nutrients & DR $(n=103)$ & $\begin{array}{c}\text { No DR } \\
(n=140)\end{array}$ & $\begin{array}{c}\text { Crude } \\
\text { OR }[95 \% \mathrm{CI}]\end{array}$ & Crude $p$ & $\begin{array}{c}\text { Adjusted } \\
\text { OR }[95 \% \text { CI }]^{1}\end{array}$ & Adjusted $p^{1}$ \\
\hline Carbohydrate $(\mathrm{g})$ & $198.0 \pm 36.3$ & $186.6 \pm 31.2$ & $1.01[1.00 ; 1.02]$ & 0.127 & $1.01[0.99 ; 1.01]$ & 0.127 \\
\hline $\begin{array}{c}\text { Complex } \\
\text { carbohydrate (g) }\end{array}$ & $91.6 \pm 18.6$ & $85.8 \pm 18.1$ & $1.02[1.00 ; 1.04]$ & 0.031 & $1.02[1.00 ; 1.04]$ & 0.031 \\
\hline Glycemic index (\%) & $87.0 \pm 18.1$ & $81.1 \pm 15.8$ & $1.02[1.00 ; 1.04]$ & 0.087 & $1.02[0.99 ; 1.04]$ & 0.087 \\
\hline Total fat (g) & $98.9 \pm 16.3$ & $105.8 \pm 13.9$ & $0.97[0.94 ; 0.99]$ & 0.009 & $0.96[0.94 ; 0.98]$ & 0.009 \\
\hline SFAs (g) & $25.4 \pm 5.1$ & $26.8 \pm 4.4$ & $0.96[0.90 ; 1.03]$ & 0.290 & $0.97[0.91 ; 1.03]$ & 0.290 \\
\hline MUFAs (g) & $50.2 \pm 11.2$ & $54.3 \pm 9.9$ & $0.96[0.92 ; 0.99]$ & 0.012 & $0.95[0.92 ; 0.99]$ & 0.012 \\
\hline Palmitic acid (g) & $15.7 \pm 2.7$ & $16.5 \pm 2.2$ & $0.90[0.78 ; 1.03]$ & 0.126 & $0.89[0.78 ; 1.02]$ & 0.126 \\
\hline Oleic acid (g) & $47.7 \pm 11.0$ & $51.7 \pm 9.7$ & $0.96[0.92 ; 0.99]$ & 0.012 & $0.95[0.92 ; 0.99]$ & 0.012 \\
\hline$\alpha$-Linolenic acid (g) & $1.1 \pm 0.2$ & $1.2 \pm 0.2$ & $0.16[0.02 ; 1.23]$ & 0.077 & $0.16[0.02 ; 1.23]$ & 0.077 \\
\hline Vitamin E (mg) & $13.9 \pm 2.9$ & $15.2 \pm 3.6$ & $0.86[0.77 ; 0.96]$ & 0.006 & $0.85[0.77 ; 0.95]$ & 0.006 \\
\hline Thiamine (mg) & $1.6 \pm 0.2$ & $1.5 \pm 0.3$ & $3.70[0.86 ; 15.84]$ & 0.078 & $3.70[0.86 ; 15.84]$ & 0.078 \\
\hline
\end{tabular}

Crude and adjusted odds ratios and corresponding 95\% confidence interval of diabetic retinopathy. Data are mean $\pm \mathrm{SD} .{ }^{1}$ Adjusted by age, sex, educational level, smoking, center, physical activity, body mass index, dyslipidemia, hypertension, diabetes duration, and glycated hemoglobin. DR, diabetic retinopathy; SFAs, saturated fatty acids; MUFAs, monounsaturated fatty acids.

\section{Discussion}

Our results suggest that the intake of total fat, MUFAs, oleic acid, and vitamin $\mathrm{E}$ is associated with the absence of DR in subjects with T1DM. In contrast, the intake of complex carbohydrates is related to the presence of DR. To our knowledge, this is the first study that has so far assessed the overall food and nutrient intake in patients with T1DM with DR. 
In terms of total fat and MUFA intake, the present results are discordant with the results of a sub-study of the DCCT trial, which found an association between the increased intake of fat, SFAs and MUFAs and the presence of DR [6]; however, these researchers did not adjust for potential confounders [5], i.e., age, sex, smoking, diabetes duration, glycated hemoglobin ( $\mathrm{HbA1c})$, body mass index (BMI), and the presence of hypertension or dyslipidemia. In addition, Roy et al. [7] did not find any association between the intake of fatty acids and the risk of 6-year progression of DR in a large cohort of T1DM subjects who consumed a high amount of SFAs, PUFAs, and dietary cholesterol. This result was in line with the results of a cross-sectional study, including well-controlled patients with T1DM and T2DM [8]. On the other hand, a protective association with higher intake of MUFAs and oleic acid was observed in a previous study in patients with T2DM performed by our group, a finding that was similar with the current results [15]. The PREDIMED trial showed that a Mediterranean diet supplemented with extra virgin olive oil (a MUFA-rich diet) and a PUFA-rich diet were related with a lower incidence of DR at follow-up in patients with T2DM [24,25]. Moreover, a cross-sectional study observed that the intake of PUFAs was a protective factor of DR in well-controlled diabetic patients [8]. However, the DCCT study found that an intake of PUFAs was related with a high risk of DR [6]; no such association was shown in another prospective study in patients with T1DM [7], a finding that is similar to our results. These two prospective studies are contrary with our results. Two meta-analyses of randomized clinical trials showed that a high-MUFA diet improves glycated hemoglobin and fasting blood glucose in patients with diabetes when compared with that of a low-MUFA diet or high-carbohydrate diet [26,27]; furthermore, MUFAs have potential benefits in the lipid profile, i.e., increasing high-density lipoprotein (HDL) serum levels in diabetic patients [27]. This result has great importance because a MUFA-rich diet may have the potential to prevent diabetic complications long-term. A recent review on the role of omega-3 in the development of DR described that $\alpha$-linolenic acid improves the progression of DR by means of various antioxidant and anti-inflammatory mechanisms in animal and human studies [28].

Interestingly, our results showed that a higher intake of carbohydrates was positively related with the presence of DR in patients with T1DM. This finding is in contrast with those of the DCCT study, which found a lower presence of DR with a high intake of carbohydrates and dietary fiber in adolescents and young adult patients with T1DM [6]. However, the other few studies that have assessed the possible association between dietary carbohydrates and DR did not find such association $[7,8,15$, 29]. In line with our results, the EURODIAB study described that a rich-carbohydrate diet derived from complex carbohydrates (not dietary fiber) produces negative effects in glycemic control and consequently a high risk of diabetic complications [30].

Our findings indicate that a higher intake of vitamin $\mathrm{E}$ is associated with a lower presence of DR; this result is due to a high intake of vegetable fats. Although a risk association between the intake of vitamin E and DR was found in a cross-sectional study including a large sample of patients with T2DM [31], some prospective and other cross-sectional studies did not confirm this association [8,32,33]. This finding should be further investigated due to controversy in the scientific studies.

In terms of alcohol consumption, we did not find any difference between the two groups; however, two cross-sectional studies performed in patients with T1DM found a positive relationship between alcohol consumption and the presence of DR [9,11]; and another cross-sectional study showed that an increased alcohol consumption was related to a lower prevalence of DR in younger-onset diabetes [12]. Therefore, we must gain more insight into this potential dietary contributing factor.

Moreover, the current study showed that a higher educational level was related with a lower presence of DR; this result is similar to the those of the EPIC study, which associated a high educational level with better dietary habits in Mediterranean countries [34]. This result points to the possible link between a healthier dietary intake and a lower prevalence of DR in diabetic subjects.

This study has some limitations. First, the association of causality between the intake of nutrients and the presence of DR cannot be established because of the cross-sectional study design. For this reason, no specific nutritional recommendations to the patients can be derived from our results. 
Furthermore, the changes in dietary habits over time cannot be determined with this study. Another limitation of our study is that we did not assess the specific knowledge of each study participant on the dietary management of diabetes and its relationship with the individual food intake. Therefore, we could not evaluate whether patients with DR had a less favorable dietary fat intake as a result of poorer adherence and/or lower knowledge of dietary measures. Moreover, this is a sub-study of a previous study designed to assess differences in the Mediterranean diet between patients with T1DM and the non-diabetic population [14]. However, this study has several strengths. This is the first study that assessed the relationship between the overall dietary intake and the prevalence of DR in patients diagnosed with T1DM. Additionally, we included a large number of participants in this multicenter study with a very well-defined sample that allowed us to establish the variability of different populations and regions. The use of the FFQ allowed us to define the proper estimation of nutrients and food intake of patients. Patients with T1DM receive nutritional education for the management of the disease over the time. In our region, the MNT is based on the nutritional recommendations of the Mediterranean diet. Therefore, we can assume that the patients had maintained the dietary habits over a long period of time; among other factors, a higher adherence to a healthy dietary pattern may help to prevent diabetes-related complications. In addition, this FFQ has been shown to be representative of the previous five-year period of the subject's food intake [35]. However, the cross-sectional design does not allow us to draw a final conclusion. Finally, we can consider that the present results could be potentially important for future research because there has been no other study that specifically assesses food and nutrient intake with the prevalence of DR in patients with T1DM.

\section{Conclusions}

In conclusion, a lower intake of total fat, MUFAs, oleic acid, and vitamin E was shown to be associated with the presence of DR. However, a lower intake of complex carbohydrates was associated with DR absence. Further research is necessary to establish associations and causality between dietary patterns and the presence of DR in this population.

Supplementary Materials: The following are available online at http:/ /www.mdpi.com/2072-6643/10/9/1184/ s1, Table S1: Daily food intake of participants according to diabetic retinopathy status.

Author Contributions: The authors' contributions were as follows: M.G.-C. and D.M. designed the research. M.G.-C., A.R.-M., M.M., N.A., X.V., A.T., E.R., N.A., and M.H. conducted the study and collected the data. M.G-C., J.R., and D.M. analyzed and interpreted the data. M.P-D., A.L., E.C., and D.M. coordinated the study. M.G.-C., J.R., and D.M wrote the paper. D.M. was the principal investigator and had the primary responsibility for the final content. All the authors read and approved the final manuscript.

Funding: This research was funded by grants PI12/00183 and PI15/00625 from Instituto de Salud Carlos III, Ministry of Economy and Competitiveness, Spain. CIBERDEM is an initiative from Instituto de Salud Carlos III (Plan Nacional de I+D+I and Fondo Europeo de Desarrollo Regional), Spain. M.G.-C. holds a predoctoral fellowship from Ministerio de Educación, Cultura y Deporte, FPU15/03005, Spain.

Acknowledgments: The authors thank the editorial support of Mònica Gratacòs in the manuscript preparation. We want to particularly acknowledge the patients, and the IGTP-HUGTP and IRBLleida (B.0000682) Biobanks integrated in the Spanish National Biobanks Network of Instituto de Salud Carlos III (PT17/0015/0045 and PT17/0015/0027, respectively), and Tumour Bank Network of Catalonia for its collaboration.

Conflicts of Interest: The authors declare no conflict of interest.

\section{References}

1. Klein, R.; Lee, K.E.; Gangnon, R.E.; Klein, B.E.K. The 25-Year Incidence of Visual Impairment in Type 1 Diabetes Mellitus. The Wisconsin Epidemiologic Study of Diabetic Retinopathy. Ophthalmology 2010, 117, 63-70. [CrossRef] [PubMed]

2. Romero-Aroca, P.; Baget-Bernaldiz, M.; Fernandez-Ballart, J.; Plana-Gil, N.; Soler-Lluis, N.; Mendez-Marin, I.; Bautista-Perez, A. Ten-year incidence of diabetic retinopathy and macular edema. Risk factors in a sample of people with type 1 diabetes. Diabetes Res. Clin. Pract. 2011, 94, 126-132. [CrossRef] [PubMed] 
3. American Diabetes Association. Lifestyle management. Sec. 4. In Standards of Medical Care in Diabetes-2017. Diabetes Care 2017, 40, S33-S43. [CrossRef]

4. Dow, C.; Mancini, F.; Rajaobelina, K.; Boutron-Ruault, M.-C.; Balkau, B.; Bonnet, F.; Fagherazzi, G. Diet and risk of diabetic retinopathy: A systematic review. Eur. J. Epidemiol. 2017. [CrossRef] [PubMed]

5. Wong, M.Y.Z.; Man, R.E.K.; Fenwick, E.K.; Gupta, P.; Li, L.-J.; van Dam, R.M.; Chong, M.F.; Lamoureux, E.L. Dietary intake and diabetic retinopathy: A systematic review. PLoS ONE 2018, 13, e0186582. [CrossRef] [PubMed]

6. Cundiff, D.K.; Nigg, C.R. Diet and diabetic retinopathy: Insights from the Diabetes Control and Complications Trial (DCCT). Med. Gen. Med. 2005, 7, 3.

7. Roy, M.S.; Janal, M.N. High caloric and sodium intakes as risk factors for progression of retinopathy in type 1 diabetes mellitus. Arch. Ophthalmol. 2010, 128, 33-39. [CrossRef] [PubMed]

8. Sasaki, M.; Kawasaki, R.; Rogers, S.; Man, R.E.K.; Itakura, K.; Xie, J.; Flood, V.; Tsubota, K.; Lamoureux, E.; Wang, J.J. The associations of dietary intake of polyunsaturated fatty acids with diabetic retinopathy in well-controlled diabetes. Investig. Ophthalmol. Vis. Sci. 2015, 56, 7473-7479. [CrossRef] [PubMed]

9. Beulens, J.; Kruidhof, J.; Grobbee, D. Alcohol consumption and risk of microvascular complications in type 1 diabetes patients: The EURODIAB Prospective Complications Study. Diabetologia 2008, 51, 1631-1638. [CrossRef] [PubMed]

10. Engelen, L.; Soedamah-Muthu, S.S.; Geleijnse, J.M.; Toeller, M.; Chaturvedi, N.; Fuller, J.H.; Schalkwijk, C.G.; Stehouwer, C.D.A. Higher dietary salt intake is associated with microalbuminuria, but not with retinopathy in individuals with type 1 diabetes: The EURODIAB Prospective Complications Study. Diabetologia 2014, 57, 2315-2323. [CrossRef] [PubMed]

11. Harjutsalo, V.; Feodoroff, M.; Forsblom, C.; Groop, P.H. Patients with Type 1 diabetes consuming alcoholic spirits have an increased risk of microvascular complications. Diabet. Med. 2014, 31, 156-164. [CrossRef] [PubMed]

12. Moss, S.E.; Klein, R.; Klein, B.E. Alcohol consumption and the prevalence of diabetic retinopathy. Ophthalmology 1992, 99, 926-932. [CrossRef]

13. Recomendaciones Nutricionales y de Educación Alimentaria en la Diabetes; ACD: Barcelona, Spain, 2013; Available online: http://www.acdiabetis.org/d_avui/docs/Document_de_consens_pdf (accessed on 8 July 2018).

14. Granado-Casas, M.; Alcubierre, N.; Martín, M.; Real, J.; Ramírez-Morros, A.M.; Cuadrado, M.; Alonso, N.; Falguera, M.; Hernández, M.; Aguilera, E.; et al. Improved adherence to Mediterranean Diet in adults with type 1 diabetes mellitus. Eur. J. Nutr. 2018. [CrossRef] [PubMed]

15. Alcubierre, N.; Navarrete-Muñoz, E.M.; Rubinat, E.; Falguera, M.; Valls, J.; Traveset, A.; Vilanova, M.-B.; Marsal, J.R.; Hernandez, M.; Granado-Casas, M.; et al. Association of low oleic acid intake with diabetic retinopathy in type 2 diabetic patients: A case-control study. Nutr. Metab. 2016, 13, 40. [CrossRef] [PubMed]

16. Bernstein, M.S.; Morabia, A.; Sloutskis, D. Definition and prevalence of sedentarism in an urban population. Am. J. Public Health 1999, 89, 862-867. [CrossRef] [PubMed]

17. Cabrera de León, A.; Rodríguez-Pérez, M.D.C.; Rodríguez-Benjumeda, L.M.; Anía-Lafuente, B.; Brito-Díaz, B.; Muros de Fuentes, M.; Almeida-González, D.; Batista-Medina, M.; Aguirre-Jaime, A. Sedentary lifestyle: Physical activity duration versus percentage of energy expenditure. Rev. Esp. Cardiol. 2007, 60, 244-250. [CrossRef] [PubMed]

18. Willett, W.C.; Sampson, L.; Stampfer, M.J.; Rosner, B.; Bain, C.; Witschi, J.; Hennekens, C.H.; Speizer, F.E. Reproducibility and validity of a semiquantitative food frequency questionnaire. Am. J. Epidemiol. 1985, 122, 51-65. [CrossRef] [PubMed]

19. Vioque, J.; Navarrete-Muñoz, E.-M.; Gimenez-Monzó, D.; García-de-la-Hera, M.; Granado, F.; Young, I.S.; Ramón, R.; Ballester, F.; Murcia, M.; Rebagliato, M.; et al. Reproducibility and validity of a food frequency questionnaire among pregnant women in a Mediterranean area. Nutr. J. 2013, 12, 26. [CrossRef] [PubMed]

20. US Department of Agriculture Agricultural Research Service. USDA National Nutrient Database for Standard Reference. Available online: https:/ / www.ars.usda.gov/ (accessed on 1 July 2018).

21. Palma, I.; Farran, P.; Cervera, P. Tablas de Composición de Alimentos por Medidas Caseras de Consumo Habitual en España; Mc Graw Hill Interamericana: Barcelona, Spain, 2008.

22. Food Standards Agency. McCance and Widdowson's The Composition of Foods, 6th ed.; Royal Society of Chemistry: Cambridge, UK, 2002. 
23. Wilkinson, C.P.; Ferris, F.L.; Klein, R.E.; Lee, P.P.; Agardh, C.D.; Davis, M.; Dills, D.; Kampik, A.; Pararajasegaram, R.; Verdaguer, J.T.; et al. Proposed international clinical diabetic retinopathy and diabetic macular edema disease severity scales. Ophthalmology 2003, 110, 1677-1682. [CrossRef]

24. Díaz-López, A.; Babio, N.; Martínez-González, M.A.; Corella, D.; Amor, A.J.; Fitó, M.; Estruch, R.; Arós, F.; Gómez-Gracia, E.; Fiol, M.; et al. Mediterranean Diet, Retinopathy, Nephropathy, and Microvascular Diabetes Complications: A Post Hoc Analysis of a Randomized Trial. Diabetes Care 2015, 38, 2134-2141. [CrossRef] [PubMed]

25. Sala-Vila, A.; Díaz-López, A.; Valls-Pedret, C.; Cofán, M.; García-Layana, A.; Lamuela-Raventós, R.-M.; Castañer, O.; Zanon-Moreno, V.; Martinez-Gonzalez, M.A.; Toledo, E.; et al. Dietary Marine $\omega-3$ Fatty Acids and Incident Sight-Threatening Retinopathy in Middle-Aged and Older Individuals with Type 2 Diabetes: Prospective Investigation from the PREDIMED Trial. JAMA Ophthalmol. 2016, 134, 1142. [CrossRef] [PubMed]

26. Schwingshackl, L.; Strasser, B.; Hoffmann, G. Effects of Monounsaturated Fatty Acids on Cardiovascular Risk Factors: A Systematic Review and Meta-Analysis. Ann. Nutr. Metab. 2011, 59, 176-186. [CrossRef] [PubMed]

27. Schwingshackl, L.; Hoffmann, G. Monounsaturated fatty acids and risk of cardiovascular disease: Synopsis of the evidence available from systematic reviews and meta-analyses. Nutrients 2012, 4, 1989-2007. [CrossRef] [PubMed]

28. Behl, T.; Kotwani, A. Omega-3 fatty acids in prevention of diabetic retinopathy. J. Pharm. Pharmacol. 2017. [CrossRef] [PubMed]

29. Horikawa, C.; Yoshimura, Y.; Kamada, C.; Tanaka, S.; Tanaka, S.; Matsunaga, S.; Hanyu, O.; Araki, A.; Ito, H.; Tanaka, A.; et al. Is the proportion of carbohydrate intake associated with the incidence of diabetes complications?-An analysis of the Japan diabetes complications study. Nutrients 2017, 9, 113. [CrossRef] [PubMed]

30. Buyken, A.E.; Toeller, M.; Heitkamp, G.; Irsigler, K.; Holler, C.; Santeusanio, F.; Stehle, P.; Fuller, J.H.; John, G.; Viberti, G.C.; et al. Carbohydrate sources and glycaemic control in type 1 diabetes mellitus. Diabet. Med. 2000, 17, 351-359. [CrossRef] [PubMed]

31. Mayer-Davis, E.J.; Bell, R.A.; Reboussin, B.A.; Rushing, J.; Marshall, J.A.; Hamman, R.F. Antioxidant nutrient intake and diabetic retinopathy. Ophthalmology 1998, 105, 2264-2270. [CrossRef]

32. Millen, A.E.; Klein, R.; Folsom, A.R.; Stevens, J.; Palta, M.; Mares, J.A. Relation between intake of vitamins C and $\mathrm{E}$ and risk of diabetic retinopathy in the Atherosclerosis Risk in Communities Study. Am. J. Clin. Nutr. 2004, 79, 865-873. [CrossRef] [PubMed]

33. Tanaka, S.; Yoshimura, Y.; Kawasaki, R.; Kamada, C.; Tanaka, S.; Horikawa, C.; Ohashi, Y.; Araki, A.; Ito, H.; Akanuma, Y.; et al. Japan Diabetes Complications Study Group Fruit Intake and Incident Diabetic Retinopathy with Type 2 Diabetes. Epidemiology 2013, 24, 204-211. [CrossRef] [PubMed]

34. Zamora-Ros, R.; Knaze, V.; Luján-Barroso, L.; Romieu, I.; Scalbert, A.; Slimani, N.; Hjartåker, A.; Engeset, D.; Skeie, G.; Overvad, K.; et al. Differences in dietary intakes, food sources and determinants of total flavonoids between Mediterranean and non-Mediterranean countries participating in the European Prospective Investigation into Cancer and Nutrition (EPIC) study. Br. J. Nutr. 2013, 109, 1498-1507. [CrossRef] [PubMed]

35. Vioque, J.; Gonzalez, L. Validez de la evaluación de la ingesta dietética. In Nutrición y Salud Pública. Métodos, Bases Científicas y Aplicaciones; Serra Majem, L., Aranceta Bartrina, J., Eds.; Masson-Elsevier: Barcelona, Spain, 2006; pp. 199-210. ISBN 84-458-1528-8.

(C) 2018 by the authors. Licensee MDPI, Basel, Switzerland. This article is an open access article distributed under the terms and conditions of the Creative Commons Attribution (CC BY) license (http://creativecommons.org/licenses/by/4.0/). 